\title{
Involvement of Glutamate Transporter-1 in Neuroprotection against Global Brain Ischemia-Reperfusion Injury Induced by Postconditioning in Rats
}

\author{
Weiqiao Zhang ${ }^{1,2}$, Yifeng Miao ${ }^{1}$, Sanquan Zhou ${ }^{2}$, Baofeng Wang ${ }^{1}$, Qizhong Luo ${ }^{1}$ \\ and Yongming Qiu ${ }^{1}$ *
}

1 Department of Neurosurgery, Ren Ji Hospital, Shanghai Jiao Tong University School of Medicine, Shanghai 200127, China; E-Mails: zhang951154@sina.com (W.Z.); 553909021@qq.com(Y.M.);kingwww629@126.com (B.W.); 13958354209@ 139.com (Q.L.)

2 Department of Neurosurgery, YuYao People's Hospital, Zhejiang Province, 315400, China; E-Mail: zsq8989@yahoo.com (S.Z.)

* Author to whom correspondence should be addressed; E-Mail: Qiuzhoub@ hotmail.com; Tel.: +86-21-58752345 ext 3748; Fax: 86-21-58394262.

Received: 17 September 2010; in revised form: 1 October 2010 / Accepted: 22 October2010 / Published: 3 November 2010

\begin{abstract}
Ischemic postconditioning refers to several transient reperfusion and ischemia cycles after an ischemic event and before a long duration of reperfusion. The procedure produces neuroprotective effects. The mechanisms underlying these neuroprotective effects are poorly understood. In this study, we found that most neurons in the CA1 region died after 10 minutes of ischemia and is followed by 72 hours of reperfusion. However, brain ischemic postconditioning (six cycles of $10 \mathrm{~s} / 10 \mathrm{~s}$ reperfusion/re-occlusion) significantly reduced neuronal death. Significant up-regulation of Glutamate transporter-1 was found after 3, 6, 24, 72 hours of reperfusion. The present study showed that ischemic postconditioning decreases cell death and that upregulation of GLT-1 expression may play an important role on this effect.
\end{abstract}

Keywords: ischemic postconditioning; brain ischemia reperfusion injury; neuroprotection; glutamate transporter 1(GLT-1) 


\section{Introduction}

Ischemic stroke is a leading cause of mortality and adult morbidity in humans. Extensive research has been conducted to find drugs that reduce injury following brain ischemia/reperfusion; however, no effective neuroprotective drugs have been found for use in clinical practice [1].

Ischemic postconditioning refers to several transient reperfusion and ischemia cycles after an ischemic event and before a long duration of reperfusion. Its cerebral protective effects have been proven in different animal models [2-5], but its mechanism has not been clarified.

Glutamate is one of the main neurotransmitters and works at a variety of excitatory synapses in the nervous system [6]. However, it is also believed to lead to glutamate excitotoxicity - an important mechanism of cell death induced by ischemic insult [7]. Many studies have proven that the extracellular concentration of glutamate is elevated to levels leading to neuronal death in ischemia.

Glutamate transporters are essential for maintaining low concentration by removing the glutamate from the extracellular space. At present, five glutamate transporters have been identified in the rat brain. These glutamate transporters, are GLAST, GLT-1, EAAT3, EAAT4 and EAAT5 [8].

Glutamate transporter-1 (GLT-1), which is the most abundant glutamate transporter in brain, plays a principal role in keeping the glutamate concentration low by removing the glutamate released at the synapse [9]. In view of the importance of GLT-1 in the maintenance of appropriate glutamate concentrations, it is reasonable to infer that GLT-1 is involved in the protective effect of brain ischemic postconditioning. However, there have been no reports regarding the role of GLT-1 in brain ischemic tolerance generated by ischemic postconditioning.

In this study, we tested the hypothesis that ischemic postconditioning reduces neuronal death by upregulating GLT-1. We used the 4-vessel occlusion (4-VO) animal model, Hematoxylin/erosin and cresyl violet staining were used to elucidate the protective effect of postconditioning in the hippocampal CA1 at 72 hours after reperfusion and hippocampal GLT-1 expression was measured at $3,6,24,72$ hours of reperfusion to demonstrate the neuronal protective mechanism of ischemic postconditioning.

\section{Results and Discussion}

\subsection{Neuropathological Evaluation of Hippocampal CA1 by Hematoxylin/Erosin Staining}

The distribution of pyramidal cells in CA1 was examined 72 hours after reperfusion. As shown in Figure 1, in the sham group, pyramidal neurons were layered and in regular arrangements with large, round, transparent, and intact nuclei (Figure 1A, 1B). In the I/R group, the neurons in the CA1 region were severely damaged, in disorderly arrays, significantly reduced in number, and characterized by pyknotic and indistinct nuclei (Figure 1C, 1D). Six cycles of 10-second/10-second of reperfusion/re-occlusion significantly decreased cell death in CA1 (Figure 1E, 1F). 
Figure 1. Neuropathological evaluation of hippocampal CA1 by hematoxylin and eosin staining. Representative photomicrographs in the hippocampal region $(\times 40)(\mathrm{A}, \mathrm{C}, \mathrm{E})$ and hippocampus CA1 (×400) (B, D, F).
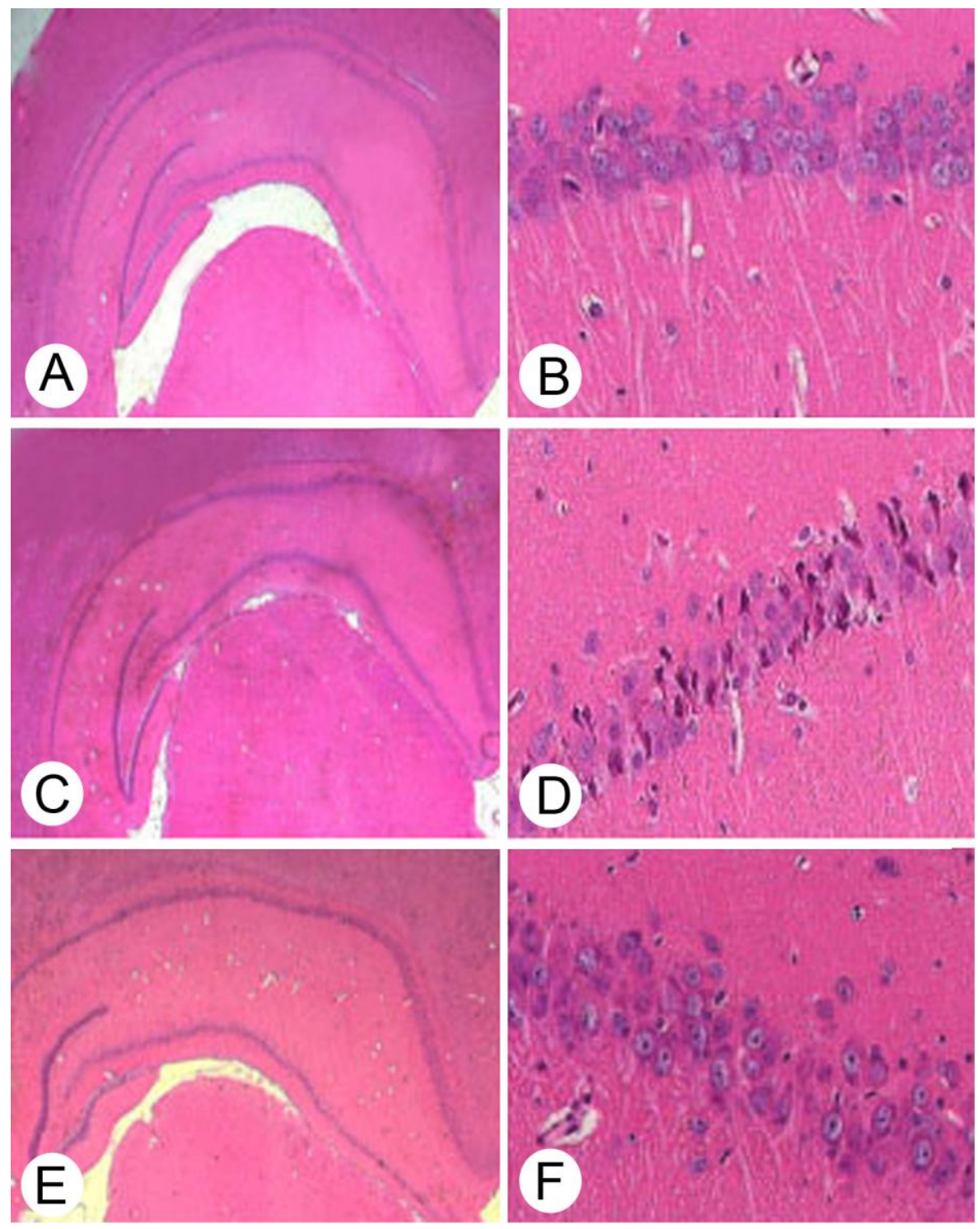

\subsection{Neuron Counts in Hippocampal CA1, as Determined by Cresyl Violet Staining}

We used the cell density to assess the effect of ischemic postconditioning in the hippocampal CA1 region by using cresyl violet staining (Figure 2). In the sham group, pyramidal cells displayed round, pale, stained nuclei. We observed that 72 hours after re-perfusion, most CA1 pyramidal cells were destroyed in $\mathrm{I} / \mathrm{R}$ group. The number of surviving neurons increased significantly in the I PostC group as compared to the $\mathrm{I} / \mathrm{R}$ group $(P<0.01)$. The numbers of surviving pyramidal cells in the hippocampal CA1 region of the I/R and I PostC groups, respectively, were $\sim 23.5 \%$ and $\sim 62.8 \%$ percent of those observed in rats treated with the sham operation (Figure 3). 
Figure 2. Neuroprotective effect of ischemic postconditioning on neuron death induced by global ischemia/reperfusion in the hippocampal CA1, as determined using cresyl violet staining. A, D: sham group; B, E: ischemia/reperfusion group; C, F: Ischemic postconditioning group. Magnification, $(\times 40)(\mathrm{A}, \mathrm{B}, \mathrm{C})$ and $(\times 400)(\mathrm{D}, \mathrm{E}, \mathrm{F})$.

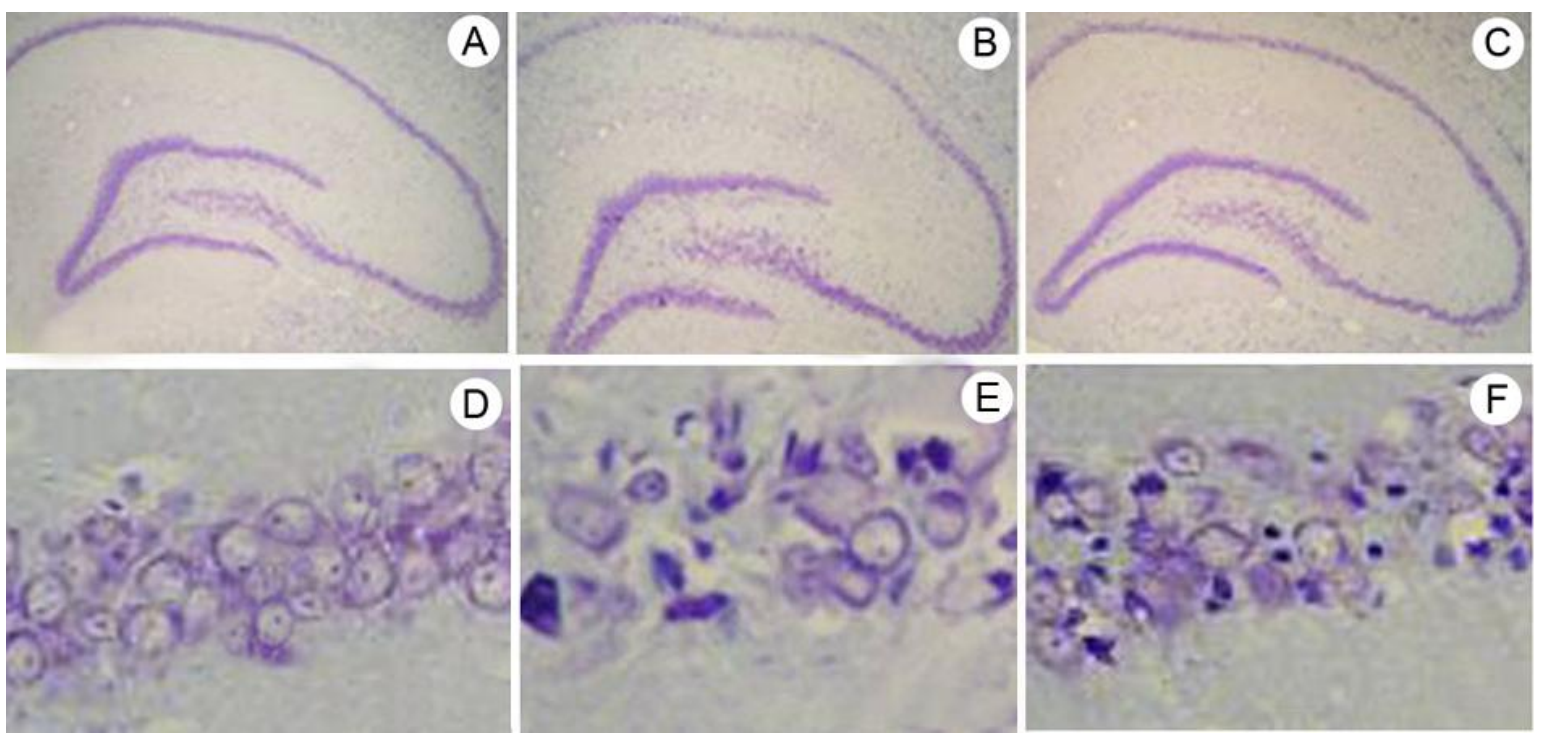

Figure 3. Histogram representing numbers of surviving neurons in CA1/mm after 72 hours of reperfusion $(\mathrm{n}=4)$. *: I/R group versus Sham group $(P<0.01)$. \#: I PostC group versus $\mathrm{I} / \mathrm{R}$ group $(P<0.01)$.

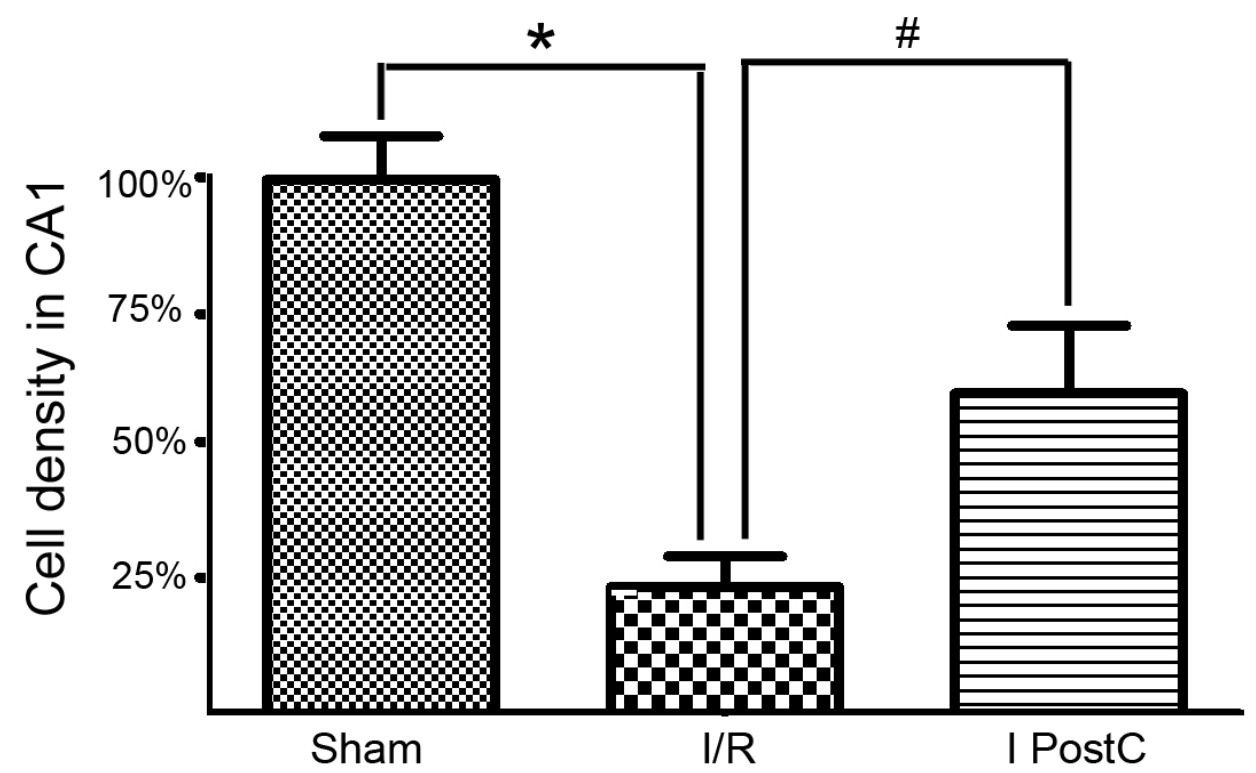

\subsection{Expression of GLT-1}

Western blot analysis was carried out to identify the expression of GLT-1 at the protein level in the hippocampus (Figure 4). The fact that GLT-1 was expressed in sham-treated animals indicated its presence in the normal rat hippocampus. At each designated time point after reperfusion, the amount of GLT-1 in both the I/R and I PostC groups increased significantly as compared with that of the sham 
group $(P<0.01)$. We observed an increase in GLT-1 protein in the I PostC group as compared with the I/R group at 3 hours, 6 hours, 24 hours and 72 hours after reperfusion, respectively. $(P<0.05)$.

Figure 4. Expression of GLT-1 protein at 3, 6, 24 and 72 hours after reperfusion. Hippocampus in the sham group, I/R group and I PostC group. *: GLT-1 of I/R group increased significantly as compared with that of the sham group $(P<0.01)$. \#: I PostC group had greater upregulation of GLT-1 compared to the I/R group at every time point $(P<0.05)$.
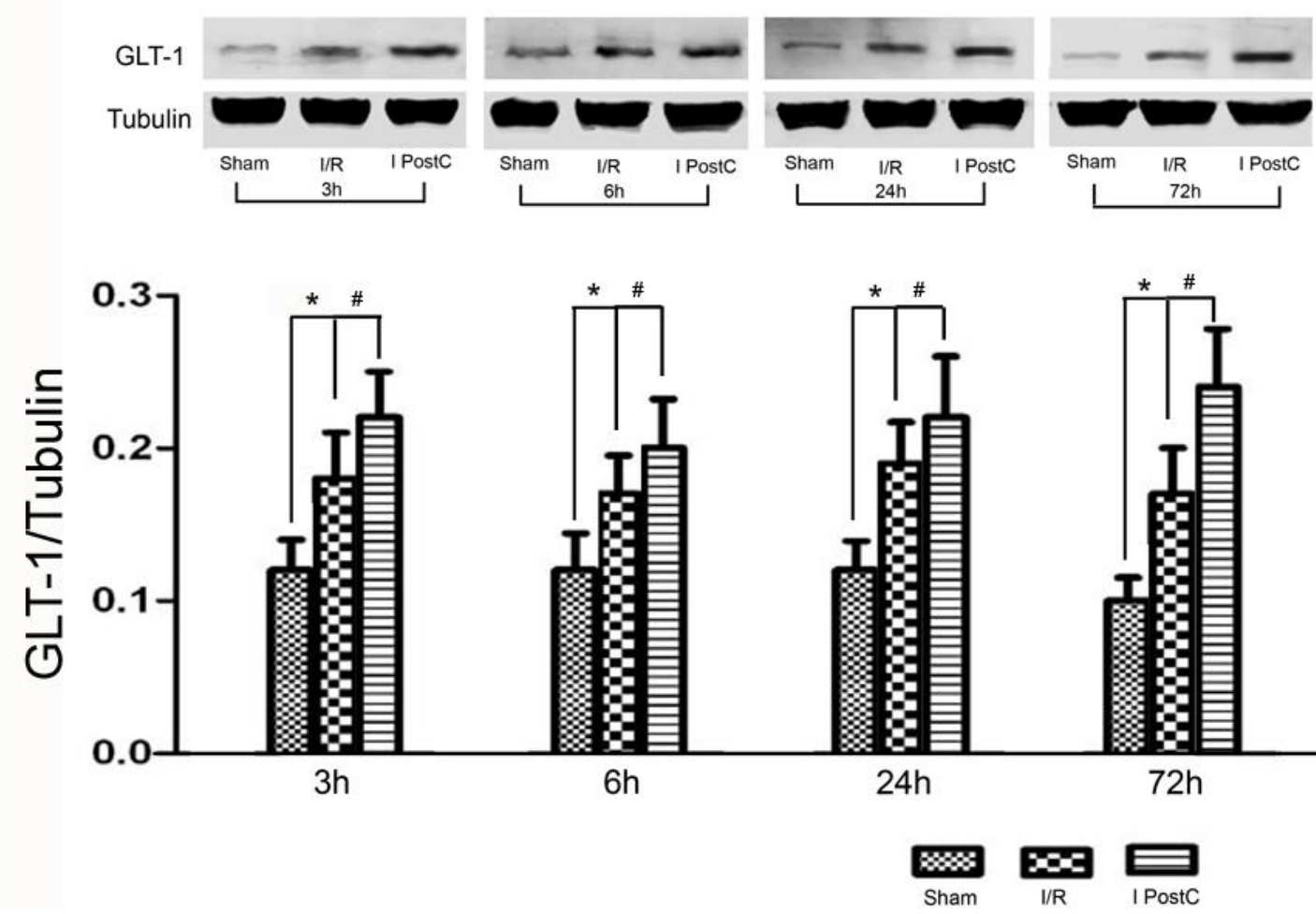

\subsection{Discussion}

Our study demonstrated that after six cycles of $10 \mathrm{~s} / 10 \mathrm{~s}$ ischemic postconditioning administered to global cerebral ischemic rats followed with reperfusion for 72 hours, the extent of neuronal cell loss was decreased significantly in the ischemic injury-prone hippocampal CA1 region. This finding suggests that ischemic postconditioning can protect neurons. We found that ischemic postconditioning can increase the GLT-1 level in hippocampus. This may be one of the mechanisms of ischemic postconditioning. Due to the upregulation of GLT-1, it is possible to decrease glutamate accumulation in the synaptic cleft and thereby reduce the overactivation of postsynaptic glutamate receptors, thus attenuating the death of neurons.

Ischemic postconditioning (I PostC) is carried out by administering several cycles of short reperfusion/ischemia at the early stage of reperfusion. Many researchers have proven that I PostC protects the brain and have been trying to study the mechanism from different perspectives. Using 4-VO global cerebral ischemic rats, Wang et al. found that I PostC can reduce neuronal death in hippocampus and increase the spatial memory of rats. This may be related to I PostC's ability to improve disturbed cerebral blood flow and inhibit the release of cytochrome c [3]. Using MCAO rats, 
Xing et al. found that I PostC can reduce infarct size, increase neurologic score, upregulate Bcl-2 and Hsp70, and inhibit caspase-3, Bax and cytochrome c. The authors speculated that brain protection by I PostC may be due to inhibition of cell apoptosis [2]. Gao et al. found that the effects of I PostC are related to changes in the Akt, MAPK and PKC signaling pathways [10,11].

The excitotoxicity of glutamate is considered to be an important reason for neuronal death after ischemia [12-14]. Therefore, reducing the amount of glutamate in extracellular fluid during ischemia would lessen the damage to neurons. Glutamate is reabsorbed mainly by glutamate transporters. Although neurons express glutamate transporter, it is usually believed that glutamate transporters, especially GLT-1, play the dominant role in maintaining extracellular glutamate level $[9,15]$.

GLT-1 could be involved in many neuropathic processes. For example, in studies on cerebral ischemia, some authors have presumed that the decrease of GLT-1 is the reason for increases in the level of glutamate [16-19]. In other studies, it was found that levels of glutamate transporter increased after ischemia. The authors considered this as a compensatory response to increased glutamate reabsorbed under these conditions [20,21]. In addition, it has been shown that GLT-1 is also involved in pathological processes such as brain trauma, neurodegenerative disease and epilepsy [22-25]. Namura et al. found that downregulation of GLT-1 can enhance brain edema after ischemia [26]. Our previous work showed that GLT-1 may play a role in ischemia-related epilepsy [27].

The role of GLT-1 in I PostC has not yet been studied. In this study, we found that hippocampal GLT-1 in I/R group increased compared to the sham group. After I PostC, a further increase of GLT-1 was found. Thus, we speculate that the upregulation of GLT-1 may be a self-protection mechanism after cerebral ischemia; I PostC can enhance the protective effect, thereby reducing neuronal damage.

\section{Experimental Section}

\subsection{Animal Groups}

All animal protocols were approved by the School of Medicine Animal Care and Use Committee of Shanghai Jiao Tong University. Eighty-four adult male Sprague-Dawley rats (220-250 g) were used for this study. The rats were randomly assigned to three groups: sham (Sham) group (3 hours, $\mathrm{n}=6$; 6 hours, $\mathrm{n}=6 ; 24$ hours, $\mathrm{n}=6 ; 72$ hours, $\mathrm{n}=10$ ); ischemia/reperfusion (I/R) group ( 3 hours, $\mathrm{n}=6$; 6 hours, $\mathrm{n}=6 ; 24$ hours, $\mathrm{n}=6 ; 72$ hours, $\mathrm{n}=10$ ) and ischemic postconditioning (I PostC) group ( 3 hours, $\mathrm{n}=6 ; 6$ hours, $\mathrm{n}=6 ; 24$ hours, $\mathrm{n}=6 ; 72$ hours, $\mathrm{n}=10$ ). Animals were housed in a 12 hours/12 hours light/dark cycle environment with free access to water and food.

\subsection{Animal Model and Surgical Procedure}

The 4-vessel occlusion (4-VO) animal model, which we have described previously, was used to establish global brain ischemia [28]. In brief, rats were anesthetized by intraperitoneal administration of $20 \%$ chloral hydrate $(350 \mathrm{mg} / \mathrm{kg})$. Both vertebral arteries were electrocauterized and the bilateral common carotid arteries were separated on day 1. On the following day, the bilateral common carotid arteries were clamped with aneurysm clips for 10 minutes to cause global brain ischemia. Rats that had lost the righting reflex, had dilated pupils, and were unresponsive to light, were used in the experiments. 
In the sham group, rats were anesthetized, both vertebral arteries were electrocauterized and the bilateral common carotid arteries were separated, but not occluded. In the ischemia/reperfusion group, rats were subjected to 10 minutes of global ischemia and then the aneurysm clip was loosened to permit reperfusion. In the ischemic postconditioning group, rats were subjected to 10 minutes of global ischemia, which was followed by ischemic postconditioning.

The ischemic postconditioning protocol was as follows: rats in the I PostC group were subjected to six cycles of 10 seconds of reperfusion and 10 seconds of ischemia by removing aneurysm clips and then occluding the clips (Figure 5).

Figure 5. Experimental protocol used to determine the effects of ischemic postconditioning (I PostC) after ischemia/reperfusion. Sham: sham-operated rats, rats were anesthetized, both vertebral arteries were electrocauterized and the bilateral common carotid arteries were separated, but not occluded; I/R: rats subjected to 10 minutes of 4-vessel occlusion (4-VO) followed by reperfusion; I PostC, rats treated with ischemic postconditioning after 10 minutes of $4-\mathrm{VO}$ by six cycles of $10 \mathrm{~s} / 10 \mathrm{~s}$ reperfusion/ischemia (at the beginning of reperfusion). Reperfusion lasted for 3, 6, 24 or 72 hours.
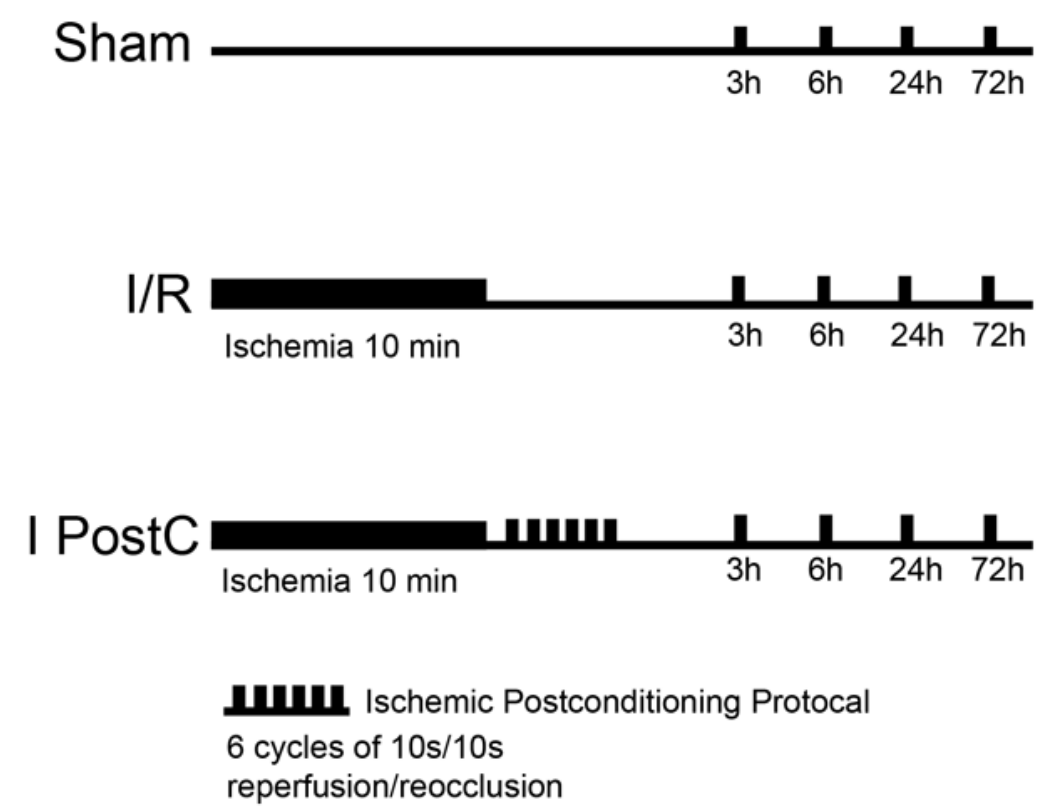

\subsection{Histological Assessment}

At 72 hours after reperfusion, rats ( $\mathrm{n}=4$ in each group) were deeply anesthetized with chloral hydrate and perfused with normal saline, followed by perfusion with $4 \%$ paraformaldehyde. The brains were quickly removed and postfixed in $4 \%$ paraformaldehyde for 24 hours. The postfixed brains were embedded in paraffin. Coronal sections $5 \mu \mathrm{m}$ thick were cut with a microtome. The sections were stained with Hematoxylin/erosin and cresyl violet, and examined with a light microscope. The cell density among hippocampal CA1, which was expressed as the ratio of survive cells per $1 \mathrm{~mm}$ length in CA1 as counted under a light microscope, was used to assess the extent of brain damage. 


\subsection{Western Blotting}

Western blotting was used to measure levels of GLT-1 protein in each animal in each group at all time points examined $(3,6,24$, and 72 hours after reperfusion; $n=6)$. The hippocampus was homogenized for $30 \mathrm{~min}$ at $4{ }^{\circ} \mathrm{C}$ with an ultrasonic wave in $200 \mathrm{~mL}$ of an RIPA lysis buffer containing: $50 \mathrm{mM}$ Tris- $\mathrm{HCl} \mathrm{pH}$ 7.4, $150 \mathrm{mM} \mathrm{NaCl}, 1 \mathrm{mM}$ EDTA, $1 \%$ Triton x-100, 1\% Sodium Deoxycholate, $0.1 \%$ SDS, add $1 \mathrm{mM}$ PMSF and proteintase inhibitor cocktail. The homogenates were then lysed in SDS buffer. Protein concentration was determined using the Bio-Rad Protein Assay Kit and the sample $(20 \mu \mathrm{g})$ was separated on Tris-glycine gel, then transferred to a nitrocellulose membrane, and immunoblotted. Blots were probed with rabbit polyclonal anti-GLT-1 (1:1000; Abcam). The blot was then incubated with IRDye $800 \mathrm{CW}$ goat anti-mouse secondary antibody (1:4000, LI-COR Biosciences, Lincoln, NE, U.S.) and bound antibodies were detected using the LI-COR imaging system. The blots were stripped and reprobed with mouse anti-tubulin (1:1000; Sigma) as a loading control. The experiments were repeated three times to obtain an average value. The band density values were calculated as a ratio of GLT-1/tublin.

\subsection{Statistical Analysis}

Data are presented as mean \pm SEM. Data analysis was performed using SAS software. Statistical significance was determined by one-way ANOVA with a Newman-Keuls post test for neuron counting. Western blot analysis was performed using two-way analysis of variance (ANOVA) followed by the Student-Newman-Keuls test. Values were considered to be significant when $P<0.05$.

\section{Conclusions}

Our study is the first to demonstrate that GLT-1 levels increased after ischemic postconditioning, which helps to further demonstrate the protective effect of I PostC on brain.

\section{Acknowledgements}

This project was supported by the Chinese National Nature Science Foundation (Grant No. 30471771) and Shanghai Science and Technology Committee (Grant No. 10140903200).

\section{References}

1. Mantz, J.; Degos, V.; Laigle, C. Recent advances in pharmacologic neuroprotection. Eur. J. Anaesthesiol 2010, 27, 6-10.

2. Xing, B.Z.; Chen, H.; Zhang, M.; Zhao, D.M.; Jiang, R.; Liu, X.H.; Zhang, S.M. Ischemic postconditioning inhibits apoptosis after focal cerebral ischemia/reperfusion injury in the rat. Stroke 2008, 39, 2362-2369.

3. Wang, J.Y.; Shen, J.; Gao, Q.; Ye, Z.G.; Yang, S.Y.; Liang, H.W.; Bruce, I.C.; Luo, B.Y.; Xia, Q. Ischemic postconditioning protects against global cerebral ischemia/reperfusion-induced injury in rats. Stroke 2008, 39, 983-990. 
4. Ren, C.C.; Yan, Z.M.; Wei, D.T.; Gao, X.W.; Chen, X.Y.; Zhao, H. Limb remote ischemic postconditioning protects against focal ischemia in rats. Brain Res. 2009, 1288, 88-94.

5. Taskapilioglu, M.O.; Alkan, T.; Goren, B.; Tureyen, K.; Sahin, S.; Taskapilioglu, O.; Korfali, E. Neuronal protective effects of focal ischemic pre- and/or postconditioning on the model of transient focal cerebral ischemia in rats. J. Clin. Neurosci. 2009, 16, 693-697.

6. Lee, S.G.; Su, Z.Z.; Emdad, L.; Gupta, P.; Sarkar, D.; Borjabad, A.; Volsky, D.J.; Fisher, P.B. Mechanism of ceftriaxone induction of excitatory amino acid transporter-2 expression and glutamate uptake in primary human astrocytes. J. Biol. Chem. 2008, 283, 13116-13123.

7. Guo, Z.H.; Li, F.; Wang, W.Z. The mechanisms of brain ischemic insult and potential protective interventions. Neurosci. Bull. 2009, 25, 139-152.

8. Ozawa, S. Role of glutamate transporters in excitatory synapses in cerebellar Purkinje cells. Brain Nerve 2007, 59, 669-676.

9. Rao, V.L.; Dogan, A.; Todd, K.G.; Bowen, K.K.; Kim, B.T.; Rothstein, J.D.; Dempsey, R.J. Antisense knockdown of the glial glutamate transporter GLT-1, but not the neuronal glutamate transporter EAAC1, exacerbates transient focal cerebral ischemia-induced neuronal damage in rat brain. J. Neurosci. 2001, 21, 1876-1883.

10. Gao, X.; Ren, C.; Zhao, H. Protective effects of ischemic postconditioning compared with gradual reperfusion or preconditioning. J. Neurosci. Res. 2008, 86, 2505-2511.

11. Gao, X.; Zhang, H.; Takahashi, T.; Hsieh, J.; Liao, J.; Steinberg, G.K.; Zhao, H. The Akt signaling pathway contributes to postconditioning's protection against stroke; the protection is associated with the MAPK and PKC pathways. J. Neurochem. 2008, 105, 943-955.

12. Nicotera, P.; Bano, D. The enemy at the gates: $\mathrm{Ca}^{2+}$ entry through TRPM7 channels and anoxic neuronal death. Cell 2003, 115, 768-770.

13. Candelario-Jalil, E. Injury and repair mechanisms in ischemic stroke: Considerations for the development of novel neurotherapeutics. Curr. Opin. Investig. Drugs 2009, 10, 644-654.

14. Taoufik, E.; Probert, L. Ischemic neuronal damage. Curr. Pharm. Des. 2008, 14, 3565-3573.

15. Romera, C.; Hurtado, O.; Mallolas, J.; Pereira, M.P.; Morales, J.R.; Romera, A.; Serena, J.; Vivancos, J.; Nombela, F.; Lorenzo, P.; et al. Ischemic preconditioning reveals that GLT1/EAAT2 glutamate transporter is a novel PPARgamma target gene involved in neuroprotection. J. Cereb. Blood Flow Metab. 2007, 27, 1327-1338.

16. Chen, J.C.; Hsu-Chou, H.; Lu, J.L.; Chiang, Y.C.; Huang, H.M.; Wang, H.L.; Wu, T.; Liao, J.J.; Yeh, T.S. Down-regulation of the glial glutamate transporter GLT-1 in rat hippocampus and striatum and its modulation by a group III metabotropic glutamate receptor antagonist following transient global forebrain ischemia. Neuropharmacology 2005, 49, 703-714.

17. Han, F.; Shioda, N.; Moriguchi, S.; Qin, Z.H.; Fukunaga, K. Downregulation of glutamate transporters is associated with elevation in extracellular glutamate concentration following rat microsphere embolism. Neurosci. Lett. 2008, 430, 275-280.

18. Rao, V.L.R.; Dogan, A.; Rothstein, J.D.; Dempsey, R.J. Decreased glial glutamate transporter GLT-1 protein levels in rat brain following transient middle cerebral artery occlusion. J. Neurochem. 1998, 70, S68. 
19. Rothstein, J.D.; Dykes-Hoberg, M.; Pardo, C.A.; Bristol, L.A.; Jin, L.; Kuncl, R.W.; Kanai, Y.; Hediger, M.A.; Wang, Y.; Schielke, J.P.; et al. Knockout of glutamate transporters reveals a major role for astroglial transport in excitotoxicity and clearance of glutamate. Neuron 1996, 16, 675-686.

20. Chu, K.; Lee, S.T.; Sinn, D.I.; Ko, S.Y.; Kim, E.H.; Kim, J.M.; Kim, S.J.; Park, D.K.; Jung, K.H.; Song, E.C.; et al. Pharmacological Induction of Ischemic Tolerance by Glutamate Transporter-1 (EAAT2) Upregulation. Stroke 2007, 38, 177-182.

21. Li, L.; Lundkvist, A.; Andersson, D.; Wilhelmsson, U.; Nagai, N.; Pardo, A.C.; Nodin, C.; Stahlberg, A.; Aprico, K.; Larsson, K.; et al. Protective role of reactive astrocytes in brain ischemia. J. Cereb. Blood Flow Metab. 2008, 28, 468-481.

22. Vanhoutte, N.; Abarca-Quinones, J.; Jordan, B.F.; Gallez, B.; Maloteaux, J.M.; Hermans, E. Enhanced expression of the high affinity glutamate transporter GLT-1 in C6 glioma cells delays tumour progression in rat. Exp. Neurol. 2009, 218, 56-63.

23. Bjornsen, L.P.; Eid, T.; Holmseth, S.; Danbolt, N.C.; Spencer, D.D.; de Lanerolle, N.C. Changes in glial glutamate transporters in human epileptogenic hippocampus: Inadequate explanation for high extracellular glutamate during seizures. Neurobiol. Dis. 2007, 25, 319-330.

24. Yi, J.H.; Hazell, A.S. Excitotoxic mechanisms and the role of astrocytic glutamate transporters in traumatic brain injury. Neurochem. Int. 2006, 48, 394-403.

25. Matute, C.; Melone, M.; Vallejo-Illarramendi, A.; Conti, F. Increased expression of the astrocytic glutamate transporter GLT-1 in the prefrontal cortex of schizophrenics. Glia 2005, 49, 451-455.

26. Namura, S.; Maeno, H.; Takami, S.; Jiang, X.F.; Kamichi, S.; Wada, K.; Nagata, I. Inhibition of glial glutamate transporter GLT-1 augments brain edema after transient focal cerebral ischemia in mice. Neurosci. Lett. 2002, 324, 117-120.

27. Lu, Z.; Zhang, W.; Zhang, N.; Jiang, J.; Luo, Q.; Qiu, Y. The expression of glutamate transporters in chest compression-induced audiogenic epilepsy: a comparative study. Neurol. Res. 2008, 30, 915-919.

28. Miao, Y.; Zhang, W.; Lin, Y.; Lu, X.; Qiu, Y. Neuroprotective effects of ischemic preconditioning on global brain ischemia through up-regulation of acid-sensing ion channel 2a. Int. J. Mol. Sci. 2010, 11, 140-153.

(C) 2010 by the authors; licensee MDPI, Basel, Switzerland. This article is an open access article distributed under the terms and conditions of the Creative Commons Attribution license (http://creativecommons.org/licenses/by/3.0/). 